\section{Do authorship disputes deter Indian medical students from pursuing research?}

Published online first on July 20, 2021. DOI: 10.20529/IJME. 2021.053

\section{Keywords: Authorship disputes, medical research, publish or perish, mentorship}

Medicine, being an ever-expanding field, makes it crucial for doctors-in-training to understand research and its methodology and translate this into their clinical practice. However, in India, the response of medical students and residents in adopting this has been sluggish, primarily owing to high levels of stress attributed to the extensive academic curriculum, hectic duty hours, and shortage of workforce that leads to an unacceptably high patient load (1). Lack of funding and mentorship programmes, difficulty in data collection and analysis, and no additional credits awarded to students for the time invested act as additional barriers to taking up research projects (2). An important but rarely discussed disincentive is the disputes regarding credits awarded to research in a publication.

Amidst the "publish or perish" academic culture, the ICMJE guidelines are infrequently adhered to. The Medical Council of India (MCl), in its circular of 2017, amended its requirements for research publications to be considered for promotion of faculty in medical colleges (3). The amendment limits credits for authorship only to the first and corresponding authors. This reform may have been intended to decrease the practice of "gift authorship"(when a faculty member is short of the required quota of papers, he/she requests a research team to include his/her name in the list of authors); but has had the opposite effect in practice (4). In postgraduate courses, students must submit a dissertation with a faculty guide and a few co-guides from the same or related disciplines. The need to comply with guidelines for periodic promotion leads to the coercion of students to add co-authors with no significant contribution, and encourages the denial of first authorship, and credit, to junior researchers whose contribution is often the most.

"Ghost authorship", defined as the failure to identify someone who is a substantial contributor to the research or written manuscript as an author, is condemned as unethical (5). On the other hand, professional medical writing assistance is an ethical and legitimate practice, permitted when appropriately acknowledged, and enhances the manuscript's quality. Ironically, "non-experts" such as medical students are subjected to being ghost authors after working on projects, with no compensation for the students' time and efforts. Such instances of denial of recognition, in the form of "gift authorship" and "ghost authorship" are unethical practices that demotivate medical students from undertaking any further research activity.

We propose some solutions to combat this negative trend:

Inclusion of credits and mentorship for research in the Indian medical curriculum

Research projects require an extensive time commitment from the mentor, especially if it is a medical students' first research project. However, it serves as an excellent mentoring opportunity for faculty to enhance students' interest in research. The inclusion of credits for research in the medical curriculum is likely to encourage students' participation.

\section{Credit matrix for research}

The International Students Surgical Network (InciSioN) designed complementary authorship guidelines that use a point-based system and a research contribution tracking spreadsheet to quantify each contributor's involvement (6). This tracker can maintain transparency and accountability amongst all contributors on a project to avoid authorship disputes. Normalising authorship discussions and managing expectations early on by faculty mentors in research projects would benefit students and junior faculty, who may lack the ability to raise these issues themselves.

\section{Collaborative multidisciplinary research}

An unexplored avenue amongst Indian medical students is collaborative research networks. These models facilitate students' participation in good quality research led by experts with different skill sets, thus widening the spectrum of learning. Publishing under a unified corporate authorship also ensures appropriate credits to all collaborators, encourages teamwork and ensures guidance for students (7). Student-led initiatives like the first student-led collaborative 'STAR SURG' research study by UK medical students, which included 1513 patients from 109 centres with each author given equal recognition (8), can provide students with experience of applied academic training

\section{Institutional policy for credits in research}

A formal institutional policy for credits in research should be advocated for in medical colleges. As the need for local datadriven solutions rises exponentially, we must provide an effective pathway to address grievances and provide students with research exposure that encourages them to continue research with their future clinical practice. 
In conclusion, research and clinical practice go hand in hand, and efforts should be focused on an inclusive approach aimed at training students as early-career researchers, equally equipped with clinical acumen and the nuances of clinical research. Given the small cadre of physician-scientists in India, a new generation of enthusiastic clinician-scientists needs to be fostered to ensure appropriate recognition and research credits.

\section{Competing interests and funding: None declared}

Rohini Dutta (corresponding author - rohdutta08@gmail.com), Medical Student, Christian Medical College, Ludhiana, Punjab, INDIA; Global Surgery Fellow, WHO Collaborating Centre for Research on Surgical Care Delivery in LMICS, Mumbai, INDIA. Deep Chakrabarti (deepchakrabarti.19@gmail.com), Senior Resident, Department of Radiation Oncology, King George's Medical University, Lucknow, INDIA; Anita Gadgil (anitgadgil@gmail.com) Head, Department of Surgery, Bhabha Atomic Research Centre Hospital, Mumbai, INDIA, Director, WHO Collaborating Centre for Research on Surgical Care Delivery in LMICs, Mumbai, INDIA.; Nobhojit Roy (nobsroy@gmail.com),WHO Collaborating Centre for Research on Surgical Care Delivery in LMICs, Mumbai, INDIA.

\section{References}

1. Chandan N, Sherkhane MS. Assessment of stress and burnout among postgraduate medical students. Natl J Community Med. 2017; 8(4): $178-82$

2. Garg R, Goyal S, Singh K. Lack of research amongst undergraduate medical students in India: It is time to act and act Now. Indian Pediatr. 2017 May 15;54(5):357-60. Doi:10.1007/s13312-017-1104-4. Epub 2017 Mar 29.

3. Medical Council of India. Amendment Notification. New Delhi, 5 June 2017[cited 2021Jan 13]. Available at www.mciindia.org/Rules-andRegulation/Gazette\%20Notifications\%20-\%20Amendments/TEQ08.06.2017.pdf

4. Bandewar SV, Pai SA. Regressive trend: MCl's approach to assessment of medical teachers' performance. Indian J Med Ethics. 2015 Oct-Dec; 12(4):192-5.Doi:10.20529/IJME.2015.052.

5. World Association of Medical Editors (WAME). Recommendations on Publication Ethics Policies for Medical Journals. Date unknown [cited 2021 Jan 06]. Available from: http://www.wame.org/ recommendations-on-publication-ethics-policies-for-medicaljournals

6. InciSioN authorship guidelines 2020. InciSioN research resources. Available from: https://incisionetwork.org/resources/

7. National Research Collaborative \& Association of Surgeons in Training Collaborative Consensus Group. Recognizing contributions to work in research collaboratives: Guidelines for standardizing reporting of authorship in collaborative research. Int J Surg. 2018 Apr; 52:355-60. Doi: 10.1016/j.ijsu.2017.12.019. Epub 2017 Dec 29.

8. STARSurg Collaborative. Impact of post-operative non-steroidal antiinflammatory drugs on adverse events after gastrointestinal surgery. Br J Surg.. 2014 Oct; 101 (11): 1413-23. DOI: http://dx.doi.org/10.1002/ bjs. 9614 . 